\title{
Tras las huellas alimentarias de nuestros ancestros "Sembrando el pasado y cultivando el presente en armonía con la Madre Tierra"
}

\author{
In the Footsteps of Food Left by Our Ancestors: \\ "Sowing the Past, Cultivating the Present in Harmony with Mother Earth"
}

\author{
Oscar Leiva Morales ${ }^{1}$ \\ Ministerio de Educación Pública \\ San José, Costa Rica \\ shidiraleiva5@gmail.com \\ Rigoberto Díaz Leiva \\ Ministerio de Educación Pública \\ San José, Costa Rica \\ moren_diaz@yahoo.es
}

\begin{abstract}
Recibido 25 de mayo de 2012 - Corregido 07 de febrero de 2013 • Aceptado 13 de marzo de 2013
Resumen. Los pueblos indígenas han enfrentado problemas alimentarios en los últimos años. La rápida destrucción de los recursos naturales, la carencia de incentivos para los productores, la globalización y los tratados de libre comercio han creado, en el pensamiento indígena, un desarraigo a la tierra. Además, ha sido difícil establecer una adecuada interrelación entre las prácticas agrícolas tradicionales y la asesoría técnica, para encauzar la economía de la comunidad con nuevas alternativas que fortalezcan los conocimientos que dejaron nuestros abuelos en cuanto al cultivo y consumo de productos agrícolas, en armonía con la naturaleza. Esta realidad, presente también en Boruca, ha traído como consecuencia, problemas económicos, sociales y culturales, aunados a una amenaza cuyos indicios ya se atisban: la pérdida de soberanía alimentaria.
\end{abstract}

Palabras claves. Comunidad indígena Boruca, soberanía alimentaria, reconversión productiva, alimentos autóctonos, Revista Electrónica Educare, Costa Rica.

Abstract. Indigenous peoples have experienced food shortages in recent years. The rapid destruction of natural resources, lack of incentives for producers, globalization and free tradeagreements have caused an uprooting from the land in the native mind. Moreover, it has been difficult to establish an adequate relationship between traditional farming practices and technical advice to put the community economy on the right track with new ways to strengthen the knowledge inherited from our grandparents on the cultivation and consumption of agricultural products, in harmony with nature. This reality, which affects the Boruca indigenous region, has led to economic, social and cultural problems, coupled with an evident threat: loss of food sovereignty.

Keywords. Boruca indigenous community, food security, productive reconversion, ative food, Educare Electronic Journal, Costa Rica.

1 Docente de idioma Boruca. Ministerio de Educación Pública de Costa Rica. Licenciado en Educación Rural y egresado de la Maestría en Educación Rural Centroamericana, Universidad Nacional de Costa Rica.

2 Docente del Ministerio de Educación Pública de Costa Rica. Licenciado en Educación Rural y egresado de la Maestría en Educación Rural Centroamericana. Universidad Nacional de Costa Rica. 
URL: http://www.una.ac.cr/educare

CORREO: educare@una.cr

\section{Introducción}

Boruca es un pueblo indígena que se ubica al sur de Costa Rica. La mayoría de su población es indígena. Actualmente cuenta con organizaciones y líderes comunales que realizan esfuerzos para implementar el conocimiento agrícola ancestral, para lo cual desarrollan intervenciones en cuanto a seguridad alimentaria y nutricional, para propiciar alternativas de vida alimenticia y saludable para esta población. Es urgente aplicar nuevas estrategias con el fin de rescatar el modo de vida productivo tradicional, pues la seguridad alimentaria autóctona es una necesidad para el buen vivir en la comunidad. Se necesita del compromiso de cada habitante para revitalizar el conocimiento de la agricultura legado por los ancestros y para avanzar en la construcción de estrategias agrícolas acordes con las particularidades de la comunidad y del contexto que la rodea.

\section{Tras las huellas alimentarias de nuestros ancestros}

Por muchas generaciones, las personas de la comunidad han cultivado la tierra, sembrando diversos productos agrícolas saludables para las familias y la comunidad, y han compartido los productos entre los habitantes del pueblo.

No podemos olvidar que antes los suelos eran muy fértiles y siempre se aseguraba una buena alimentación. Se trabajaba la tierra de acuerdo con los conocimientos que se transmitían oralmente. Ahora, con el paso del tiempo, surgen nuevos problemas para los que trabajan la tierra con sus propias manos y de forma artesanal.

Los medios de comunicación y el comercio nos presentan la alimentación de una forma fácil. Hacen pensar que es mejor comprar los productos que sembrarlos y producirlos, pues esto implica muchos costos, desde el momento en que se prepara el terreno hasta la cosecha de los productos que nutren las familias.

Esto permite ver y pensar que la alimentación de un pueblo no es simple, más bien, es un conjunto de elementos complejos. Las estrategias inadecuadas de poder se manifiestan a través de políticas económicas -incluidas las agrícolas y alimentarias- que acarrean profundas fragmentaciones sociales, las cuales son semilla del individualismo, del consumismo despiadado, de la soledad y de la incomunicación consigo mismo y con las personas que nos rodean, en tiempos de la tecnología de punta.

\section{El alimento y la comunidad}

Para los pueblos indígenas lo significativo de los alimentos no es el número de ingredientes o la calidad de ellos, sino su capacidad de vincular a la población entre sí, al vecino y a toda la población, a la colectividad. Es la capacidad de comunicar, intercambiar productos, compartir momentos importantes para la familia y para el pueblo, como las tamaleadas y chichadas. Antes, una labor no se podía realizar sin que mediara el intercambio de trabajo y de comida entre familias, parientes y amigos. Su sentido y significado están en relación con la 
interpretación del trabajo comunitario y compartido. Al igual que la comida, la música y la fiesta formaban parte de las actividades más relevantes.

Desde una perspectiva histórica, se piensa "que en tiempos precolombinos, la cosmovisión brunca reflejaba una relación de reciprocidad entre el ser humano y la madre tierra y que se expresaba en forma similar a la de otros pueblos indígenas" (Instituto de Estudios de las Tradiciones Sagradas de Abia Yala [IETSAY], 2001, p. 16).

El alimento de todos los días implica una relación directa con la tierra, porque de ahí es de donde se adquiere. Su obtención tiene un profundo sentido humano y espiritual para nuestro pueblo, pues muchos conocimientos del cultivo y producción de los granos y semillas tiene raíces ancestrales: estas se deben fortalecer para las generaciones futuras.

No solo del pan diario que nos da la tierra con que nos nutrimos y vivimos. También la sensación de los pies desnudos sobre la tierra, de la lluvia que hace crecer los cultivos sembrados; la visión de la montaña que da el aire puro y fresco que respiramos, del sol que nos alumbra, de la luna que nos dice que es momento de sembrar y recolectar las cosechas, del río y el arroyo como fuentes de vida, del paisaje y los pájaros que cantan; la vivencia del ritual del baile de los diablitos, de la conversación con las personas mayores sobre las leyendas y tradiciones de nuestros antepasados...

No por ello el régimen alimenticio de cada pueblo deja de tener una base terrenal concreta. El rancho de palma y la troja que sirven para la recolección de frutos y semillas son la combinación de trabajo y meditación, propicios para la comunicación y el intercambio, por la complementación comunitaria, el trueque entre pueblos y familias. Hoy, en algunos casos, todavía se practican estas actividades. Por ejemplo, cuando escasea la comida, si alguien tiene cosecha, invita a otras personas a la chagua (terreno cultivado con diferentes productos agrícolas de la zona) y les ofrece parte de lo cosechado para llevar a su casa, lo que luego compartirán con su familia.

Además, la alimentación juega otro papel, así “(...) un pueblo que sabe comer, pone sus platillos en el arte y en las manifestaciones populares más diversas (Amador, 2008, p. 59).

La visión y forma de pensar del indígena es apegada a la tierra y a los recursos naturales, porque aquí se encuentra el potencial alimenticio, con sus particularidades y esto constituye una manera de enfrentar la vida cotidiana, una situación compartida entre cada uno de nuestros pueblos.

Desde la conquista hasta la actualidad, no se han suprimido nuestros ricos y variados platillos alimenticios ancestrales como: el cubrív3, el oscua ${ }^{4}$, el cubújgua 5 . Sin embargo, la sociedad de poder jerarquizó las costumbres alimentarias y el trigo pasó a representar la cima del poder; mientras que el maíz y nuestra chicha representaban la ignorancia y la pobreza. Una visión muy reducida por parte del invasor, pues para ellos solamente eso es lo que sabemos

3 Maíz y frijol tierno con carne, cocinado.

4 Hongo blanco en forma de oreja, comestible.

5 Atol de maíz sancochado y molido. 
URL: http://www.una.ac.cr/educare

CORREO: educare@una.cr

comer, a pesar de la existencia de una dieta diversificada complementada con productos autóctonos que depara la madre naturaleza.

Es importante destacar que el régimen alimentario de cada pueblo indígena es inseparable de su visión del mundo (cosmovisión y cosmovivencia) y de los modos de compartir. Era la única forma de sobrevivir y siempre, hasta la actualidad, se siguen venciendo los obstáculos sociales y culturales.

El maíz para nuestros antepasados era la principal fuente alimentaria. Se le daba múltiples usos. Lo comían cocinado cuando estaba tierno, cuando estaba endureciendo lo comían asado al fuego. Se hacía atol, tortillas, tamales con carne y con frijoles. Entre sus principales usos estaba fabricar la chicha. Se le ha dado una utilidad particular a la chicha en el pueblo de Boruca, especialmente para compartir en los eventos y actividades importantes como: la Fiesta de los Diablitos, la Mura ${ }^{6}$, las Juntas, los cumpleaños. El consumo de estas bebidas se realizaba de manera restringida, cuando la comunidad necesitaba socializarse para desarrollar algún evento importante.

Actualmente son muchos los problemas que se enfrentan en la producción agrícola. Los bruncas no estamos exentos de ellos. Se necesita asistencia técnica en el campo agrícola para un mejor aprovechamiento de los suelos, para producir más, con menos costos y responder a las necesidades de la población, así como mayor apoyo a las actividades agrícolas que son solamente para el autoconsumo.

Por esta zona, hay temporadas en que algunos productos se vuelven relevantes, como el café y el tiquizque. Sin embargo los agricultores mencionan que los costos de mantenimiento son tan altos, que al final de la cosecha no se puede recuperar la inversión. En ocasiones se siembra ayote, y cuando el agricultor desea venderlo, hay una sobreoferta del producto que resulta pagado a menor precio, con menos ganancias para el productor.

Los altos costos de los abonos y químicos que se utilizan en la producción de los granos básicos ocasionan el abandono de la agricultura por parte de los indígenas; se crea, así, más dependencia de los productos vendidos en los supermercados y pulperías.

Además, la alimentación juega otro papel, así "un pueblo que sabe comer, pone sus platillos en el arte y en las manifestaciones populares más diversas (Amador, 2008, p. 59).

6 Los diablitos recuerdan la guerra entre españoles e indígenas. Se celebra cada año, del 31 de diciembre al 2 de enero. En esta festividad, el toro representa el invasor español y los diablitos representan los indígenas. Al final matan al toro como representación heroica: los indígenas vencen a los españoles. La fiesta de La Mura se celebra todos los años, los días 7, 8, 9 de diciembre en alusión a la Virgen Purísima. Cuentan los mayores que esta celebración hace referencia a unos negros que llegaron a Boruca con un tambor y un pito con el cual bailaban. Tenían acento jamaiquino, poco entendible. Esta tradición quedó para recordar el acontecimiento y por eso en ella los participantes se pintan de negro. 


\section{Fortalecer la estructura interna de los pueblos}

La educación en las áreas rurales es una herramienta para que la población pueda fortalecer sus estructuras organizativas y comunales, y contribuir en la construcción de su propio desarrollo social y cultural, superando las desigualdades sociales con nuevas alternativas para mitigar la pobreza en las zonas más olvidadas socioeconómicamente.

Revitalizar la estructura interna, en lo referente al sustento diario, como la economía de la convivencia. Las formas de organización familiar y comunal, sus sistemas de valores trasmitidos oralmente en su propia lengua, sus formas de comunicación, solidaridad y convivencia comunitaria contribuyen con el desarrollo de las prácticas agrícolas autóctonas. Por su parte, es importante considerar las formas de tenencia y cultivo de la tierra de las familias y grupos agrícolas, con la aplicación de estrategias adecuadas para el manejo de los recursos naturales y del medio ambiente. Fortalecer la estructura interna entre los pueblos indígenas y su régimen alimenticio, en el presente, significa crear una soberanía alimentaria específica y asegurar una vida próspera para nuestros niños y jóvenes.

\section{Alimentación y salud}

(...) La gente era saludable muy pocos se enfermaban y si se enfermaban, se curaban con medicina natural (...) (IETSAY, 2001, p. 24)

La buena alimentación significa salud preventiva y curativa. Cada pueblo trata de dar respuesta a las enfermedades y es heredero de un pensamiento milenario por la adaptación a la naturaleza. Es parte de la sostenibilidad alimentaria y de convivencia con la naturaleza: no cansar la tierra, dejarla descansar, rotar los cultivos, no cortar los árboles, solo los que se necesitan, no cazar la venada, no pescar en tiempos de reproducción y mucho menos envenenar los ríos, no cortar los árboles en luna nueva porque se secan, sembrar en menguante porque hay más abundancia en la cosecha.

\section{Los tiempos de buena cosecha}

Un día un grupo de campesinos comentaban en el pueblo de Boruca acerca de las tierras fértiles que había en la zona. No había necesidad de abonar ni fumigar para alcanzar una buena producción de los granos básicos. Todas las verduras, hojas, semillas y frutas que se comían eran saludables, pues no llevaban ningún químico considerado como veneno para nuestro cuerpo.

Ahora es más difícil sembrar la tierra que está cansada, además del poco respaldo que tienen los que la cultivan. 
URL: http://www.una.ac.cr/educare

CORREO: educare@una.cr

En la actualidad, el mundo rural se caracteriza por una reducción del empleo agrícola, paralelo a una mayor presencia de actividades tradicionalmente vinculadas al medio urbano, como el comercio y los servicios, una apertura a la industria (Aguilar et al., 2003).

A los que siembran el maíz y frijol les cuesta salir adelante por los altos costos de los insumos agrícolas. Si los campesinos logran una buena cosecha de arroz, el Estado importa arroz de otros países y tienen que venderlo barato para no perder la producción. Si se viene un temporal o una sequía, se pierden los cultivos y quedan con muchas deudas y las familias sufren mucho.

En una conversación informal, los campesinos expresaban acerca de la situación actual de la crisis económica que viven diariamente las familias ¿Qué pasaría si tuviéramos un presidente o un ministro de agricultura que haya tenido que trabajar bajo el sol en el campo, que sepa lo duro que es trabajar la tierra? La vida en el campo es dura, para sobrevivir con la familia, hay que trabajar con poco apoyo del gobierno.

\section{Pérdida de la soberanía alimentaria}

Un fenómeno fundamental que se genera como efecto del sentido de participación y de integración, es que la comunidad convence de que sus problemas no van a resolverse desde afuera. (Castro, 2007, p. 25)

En el ámbito nacional, los pequeños productores pueden vender sus cosechas gracias a iniciativas de algunos medios de comunicación, al menos una parte, otro resto se comercializa a través de iniciativas de comercio solidario. Sin embargo, pareciera que la política del Ministerio de Agricultura es la de acabar con la producción campesina rural. Para los gobernantes son importantes los agronegocios basados en monocultivos, repletos de agroquímicos y de negativos impactos ambientales y sociales.

Por ejemplo, el frijol que se importa de otros países tiene un precio más bajo para el consumidor que el frijol producido por un pequeño agricultor. Esto, debido a las políticas de comercio exterior que favorecen la importación de productos traídos de otros países del mundo.

Hay impactos consecuentes, como la desaparición de las familias campesinas que cultivan productos agrícolas autóctonos, para ser consumistas de los alimentos que nos venden las cadenas de comercio. Si los agricultores no son eficientes y de calidad, es porque hace falta el apoyo necesario para producir.

Vamos hacia un camino de pérdida de la seguridad alimentaria autóctona, si no se educa a nuestra niñez y juventud. Estamos en el momento oportuno para recuperar la producción de alimentos en pequeña escala y basados en la diversidad, pero también del control, como pueblo agrícola, sobre nuestra alimentación y políticas gubernamentales que favorezcan la agricultura. 
Hoy en día se aprueban tratados de libre comercio con otros países. Se siguen inventando argumentos para equilibrar los efectos negativos de los tratados. Sin embargo, la producción nacional va desapareciendo lentamente. Ante esta situación es importante que en el campo aparezcan redes de hombres y mujeres, para la promoción de una economía solidaria y la comercialización de lo producido basado en la biodiversidad. Es necesario proteger las microempresas de familias y agrupaciones, y realizar nuevas propuestas para apoyar al pequeño agricultor.

“El problema es que nosotros vemos en el gobierno solo contrapresión actúa; debería darles vergüenza tener al agro tan abandonado, nunca se ha visto una crisis así y no es por un asunto de plagas o terreno (...)" (Campos, citado en Lanzas, 2012, Sólo contra presión actúan, párr. 1); es por la falta de voluntad política para aplicar la salvaguarda. Dentro de la producción agrícola el campesino rural queda por fuera, pues las grandes empresas tienen la posibilidad de sembrar grandes extensiones de terreno, ya que tienen sus propias fincas y ayuda gubernamental (Campos, citado en Lanzas, 2012).

\section{La participación femenina desde un enfoque de género}

Aquellas mujeres que vivían en el campo, comunes y corrientes, eran como hombres también. Ellas se daban a la tarea de ayudarle al hombre a cosechar(...) uno de los trabajos era el pilado de arroz. (Amador, 2008, p. 95)

En el campo, la participación femenina es evidente y decisoria. Queda demostrado que las mujeres no solo pueden apoyar y participar en los procesos de desarrollo, sino que además poseen capacidad e interés en la aplicación de diversos proyectos.

Hay mujeres en la comunidad con terrenos familiares, donde siembran productos agrícolas y se ayudan entre sí, se comparten los trabajos, y conocimientos para enseñar, mantener y asegurar la alimentación con productos originales, entre los que se destacan el frijol boruca, el ñame, el banano, la yuca, el plátano. Practican la rotación de cultivos de granos básicos. Además incorporan la crianza de animales domésticos y aves de corral.

\section{Socializando la agricultura para el futuro}

Nuestros abuelos y líderes comentan que es importante proporcionarles a los niños y jóvenes las herramientas necesarias para la agricultura, preparando y convirtiendo el terreno de cultivo en un verdadero laboratorio, donde adquieran conocimientos relacionados con el cultivo de la tierra, como lo hacían nuestros ancestros. 
URL: http://www.una.ac.cr/educare

CORREO: educare@una.cr

De las prácticas surge el conocimiento y si los siembros se trabajan utilizando técnicas orgánicas, los niños y niñas aprenderán a producir de una forma más sana, segura y amigable con el ambiente, y tendrán experiencias que aplicarán a lo largo de toda su vida productiva.

Los grupos de docentes, el alumnado y los padres y madres de familia deben tomar en cuenta algunos factores para que los individuos logren el éxito en el campo agrícola, cultural y académico. Como experiencia demostrativa, deben usarse cultivos de siembra directa para que sea fácil trabajar, además, deben buscarse cultivos de ciclo corto como el maíz, el frijol, la yuca, el frijol de palo para que puedan ser cosechados durante el ciclo escolar.

Debe tenerse en cuenta la participación de los alumnos y las alumnas en actividades como: preparación, siembra, abonado, riego, cosecha y consumo de los alimentos cosechados.

El estudiantado debe aprender a preparar algún tipo de abono y plaguicida orgánico para el manejo de plagas y enfermedades de mayor frecuencia en la agricultura orgánica, de manera más sana y de la mano con el medio ambiente.

Es importante consuman alimentos que se siembran en el huerto escolar y lleven a sus casas una muestra de los productos cosechados ahí, para proporcionar, así, a las familias el conocimiento de los productos autóctonos y la seguridad alimentaria que este conocimiento conlleva.

Por lo tanto, debe realizarse una reconversión productiva para nuestro pueblo, es decir, obtener una mayor dinámica en el crecimiento de los productos autóctonos para la seguridad alimentaria. En ello se debe tener siempre presente que nuestros ancestros gozaban de una buena alimentación, basada en la práctica de consumir productos agrícolas cultivados por ellos mismos en la comunidad. Esto también generaba que ciertos productos se vendían en los pueblos cercanos a la costa pacífica, lo cual generaba recursos económicos a las familias y creaba una independencia alimentaria.

\section{Conclusión}

Hace años los trabajos eran compartidos, se laboraba en forma solidaria a través de las juntas o mediante un sistema llamado mano vuelta que, según los indígenas, figuraba como un sistema productivo de reciprocidad que consistía en trabajar "hoy con usted y mañana conmigo". Todos tenían arroz, maíz, cerdos y gallinas para el consumo. No había comerciante que los comprara, pero se compartían los alimentos, si alguien carecía de ellos.

Todavía hoy los amigos y vecinos del pueblo comparten comidas tradicionales como iguana, pescado, camarones, productos derivados del maíz, frutas, semillas, hojas, hongos, y en las actividades escolares y comunales, colocan en las mesas de reuniones comidas del tiempo pasado para recordar la tradición de compartir y convivir de manera armoniosa entre vecinos. 
Hace algunas décadas, en la comunidad boruca, nuestros ancestros se alimentaban con productos autóctonos y libres de contaminación, siguiendo reglas ancestrales, aseguraban el uso racional de los productos sin causar daños al medio ambiente y mantenían una dieta alimenticia muy saludable, situación que se debe poner en práctica nuevamente.

Con la llegada de la electricidad y el intercambio, fueron adquiriendo otra tradición culinaria, que complementó los productos alimenticios propios basados en el maíz, el frijol, el cacao, el pejibaye y el banano.

La amenaza de los nuevos modos productivos obliga a la comunidad de Boruca a actuar, a partir de su propia identidad, para recuperar la soberanía alimentaria. Esta gestión requiere del concurso de todos los sectores comunitarios, incluyendo mujeres y jóvenes, para determinar, en conjunto, nuestro propio porvenir.

\section{Referencias}

Aguilar, M. E., Angulo, L., Cerdas, Y., Céspedes, E., Monge, M. E., Ovares, S., Solano, J. y van Kampen, P. (2003). Un acercamiento a la educación general básica de las zonas rurales de seis paises centroamericanos. Un estudio exploratorio. Heredia: EFUNA.

Amador, J. L. (2008). Historia y tradición en Potrero Grande. Un pueblo costarricense de origen chiricano-panameño. San José, Costa Rica: EUNED.

Castro, O. O. (2007). Autogestión de las organizaciones locales. Una herramienta para el desarrollo comunitario. Comayagua, Honduras: Universidad Nacional.

Instituto de Estudios de las Tradiciones Sagradas de Abia Yala (IETSAY). (2001). Narraciones bruncas: Boruca y curré. San José, Costa Rica. Fundación coordinadora de pastoral aborigen.

Lanzas, M. S. (3 de mayo de 2012). Arroceros preparan marcha. Diario Extra. Recuperado de http://www.diarioextra.com/2012/mayo/03/nacionales18.php

\section{Cómo citar este artículo en APA:}

Leiva, O. y Díaz, R. (2013). Tras las huellas alimentarias de nuestros ancestros "Sembrando el pasado y cultivando el presente en armonía con la Madre Tierra". Revista Electrónica Educare, 17(2), 81-89. Recuperado de http://www.revistas.una.ac.cr/index. php/EDUCARE/issue/current

Nota: Para citar este artículo en otros sistemas puede consultar el hipervínculo "Como citar el artículo" en la barra derecha de nuestro sitio web:

http://www.revistas.una.ac.cr/index.php/EDUCARE/index 